\title{
Lactobacillus rhamnosus GG: An Overview to Explore the Rationale of Its Use in Cancer
}

\author{
Giuseppe L. Banna ${ }^{1 t}$, Francesco Torino ${ }^{21}$, Francesco Marletta ${ }^{3}$, Maria Santagati ${ }^{4}$, \\ Rossella Salemi ${ }^{5}$, Elisa Cannarozzo ${ }^{5}$, Luca Falzone ${ }^{5}$, Francesco Ferraù ${ }^{6}$ and \\ Massimo Libra ${ }^{5 *}$
}

'Division of Medical Oncology, Cannizzaro Hospital, Catania, Italy, ${ }^{2}$ Department of Systems Medicine, Chair of Medical Oncology, Tor Vergata University of Rome, Rome, Italy, ${ }^{3}$ Division of Radiotherapy, Cannizzaro Hospital, Catania, Italy, ${ }^{4}$ Department of Biomedical and Biotechnological Sciences, Section of Microbiology, University of Catania, Catania, Italy, ${ }^{5}$ Department of Biomedical and Biotechnological Sciences, Laboratory of Translational Oncology and Functional Genomics, Section of General and Clinical Pathology and Oncology, University of Catania, Catania, Italy, ${ }^{6}$ Division of Medical Oncology, San Vincenzo Hospital, Taormina, Italy

OPEN ACCESS

Edited by:

Cesare Mancuso,

Università Cattolica del Sacro Cuore,

Italy

Reviewed by:

Nadiya V. Boyko,

Uzhhorod National University, Ukraine

Atte Von Wright,

University of Eastern Finland, Finland

*Correspondence:

Massimo Libra

m.libra@unict.it

${ }^{t}$ These authors have contributed equally to this work.

¥Shared senior authorship

Specialty section:

This article was submitted to

Experimental Pharmacology and Drug

Discovery,

a section of the journal

Frontiers in Pharmacology

Received: 13 June 2017

Accepted: 21 August 2017

Published: 01 September 2017

Citation

Banna GL, Torino F, Marletta F, Santagati M, Salemi $R$,

Cannarozzo E, Falzone L, Ferraù $F$ and Libra M (2017) Lactobacillus rhamnosus GG: An Overview

to Explore the Rationale of Its Use

in Cancer. Front. Pharmacol. 8:603.

doi: 10.3389/fphar.2017.00603
Cancer is the second leading cause of death in the western world. In the era of precision medicine, a significant number of cancer patients can be cured with several anti-cancer therapeutic regimens. However, therapy failure may be caused by treatment side effects, such as diarrhea, especially occurring in patients with gastrointestinal or pelvic malignancies. In particular, diarrhea is one of the most frequent gastrointestinal toxicity during cancer treatment and it can result from nearly bot chemo- and radiotherapeutic strategies currently used. Diarrhea has a serious impact on patients' quality of life and treatment dosing and schedule modification due to its severity can negatively influence treatment outcomes. In this context, probiotics may play an interesting role in several human diseases with an inflammatory bowel involvement and, among these, Lactobacillus rhamnosus GG (LGG) is one of the most characterized and utilized. In particular, LGG is able to reverse intestinal dysbiosis and moderate diarrhea. Moreover, preclinical studies have documented its effects in reducing chronic inflammation associated with cancer development. This review summarizes the preclinical results of LGG on cancer cells proliferation and tumor invasion as well as the potential role of LGG use in cancer patients for the prevention and management of diarrhea associated with cancer treatment. Overall, these encouraging data support further investigation on the use of LGG in stratified patients undergoing specific therapeutic protocols, including chemotherapy and pelvic radiotherapy, in order to reduce the development of severe diarrhea and thus improve the adherence to the therapy and patients' quality of life.

Keywords: Lactobacillus rhamnosus GG, probiotics, diarrhea, cancer, chemotherapy, immunotherapy, radiotherapy

\section{INTRODUCTION}

Cancer remains the second leading cause of death in the western world (Siegel et al., 2017). Currently, a significant fraction of cancer patients can benefit from several types of therapeutic strategies. However, toxicities associated to chemotherapy and radiotherapy can negatively impact treatments responses by altering treatment dosing and schedules as well as affecting patient's quality 
of life (Banna et al., 2010). Among the gastrointestinal adverse events (AEs) diarrhea is a frequent and potentially fatal toxicity associated with anti-cancer treatment. (Gibson and Stringer, 2009) In fact, diarrhea of any grade occurs in $30-87 \%$ of patients receiving chemotherapy and in $20-49 \%$ of patients receiving pelvic radiotherapy. The incidence of severe and life-threatening (grade 3/4) diarrhea ranges from 20 to $40 \%$ in patients receiving combined chemoradiotherapy (Benson et al., 2004).

In this context, an important role may be played by probiotics, which include lactic acid bacteria (LAB). Probiotics represent the most common supplement therapy used during intestinal dysbiosis. In 2002, the Joint Food and Agriculture Organization of the United Nations/World Health Organization (FAO/WHO) working group defined probiotics as "live microorganisms, which when consumed in adequate amounts, confer a health effect on the host" (FAO/WHO, 2002). Accordingly, the best wellknown benefit of probiotics is the restoration of the normal microbiota during the antibiotic therapy (Guandalini, 2011). Moreover, probiotics may offer several prophylactic functions such as improved microbial balance and immuno-enhancement of the gastrointestinal tract (GIT) (Gill et al., 2000; Ashraf and Shah, 2014).

Gut colonization is fundamental for the complete tropism of the small bowel and the colorectal tract, to achieve full maturation of both local and systemic immune defenses, to protect against the breaching of opportunistic infections through the epithelial barrier, as well as to hone the balance between immune response and tolerance toward non-pathogenic agents (Sekirov et al., 2010).

Although each individual have a distinctive microbiota (The Human Microbiome Project Consortium, 2012), the gut bacterial population is mainly addressable to Firmicutes and Bacteroidetes (Ley et al., 2006) and it can greatly differ in composition and abundance across the intestinal tract, especially comparing the small intestine to the colon (Zoetendal et al., 2008). Unfortunately, changes in each unique gut microbiota community may induce the condition named dysbiosis as consequence of balance loss among commensal, mutualist, and opportunist/pathobiont microorganisms (Figure 1). Commensals and mutualists reside in the host gut by, respectively, showing no known effect and establishing a positive relationship for both the parts. Opportunists and pathogens, in adequate amount or under specific conditions may express their virulence and establish an inflammatory response (Kamada et al., 2013; DeGruttola et al., 2016; Lin and Zhang, 2017). Major features of the onset of dysbiosis are frequently displayed by: reduced number of beneficial microorganisms, increased density of opportunistic colonies and loss of heterogeneity in microbial composition. (DeGruttola et al., 2016). Several factors may trigger changes in each unique composition, these have been related to environmental factors, including diet, resulting in epigenetic changes (Sekirov et al., 2010; Hullar and Fu, 2014; Krautkramer et al., 2016). Unfortunately, dysbiosis is commonly associated with gut diseases characterized by symptoms like nausea, abdominal pain, discomfort, bloating, and diarrhea (Pace et al., 2015; DeGruttola et al., 2016; Lin and Zhang, 2017).
Diarrhea is one of the most common adverse event described during the course of cancer treatments (Mego et al., 2015; McQuade et al., 2016). Depending on its severity it has a serious impact on patients quality of life, on the adherence to treatment and when not properly managed con lead to life-threatening clinical conditions. (Benson et al., 2004; Davis and Milner, 2009; Pace et al., 2015). Several mechanisms are responsible for anti-cancer treatments associated diarrhea. Radiation therapy is a backbone in the treatment of both primary and recurrent pelvic and gastrointestinal malignancies. Despite the advantage of radiotherapy in these settings are widely accepted the damage of normal tissues located within the radiation therapy field can be severe. Abdominal and pelvic irradiation may, at first, trigger extensive crypt loss and direct apoptosis, especially in the region of quiescent stem cells (Ciorba et al., 2012). Gradually, radiation insults lead to the accumulation of reactive oxygen species (ROS), microvascular sclerosis, and ischemia of the tissue and loss of stem cells (Denham and Hauer-Jensen, 2002; Hatoum et al., 2006; Andreyev et al., 2012; Stansborough et al., 2017). The reduced number of cells and crypts, together with microvascular damage are inevitably bound to the impaired functionality of the GIT, especially reducing the absorptive area and leading to the development of diarrhea. These initial alteration amounts to an acute enteritis that can gradually evolve into a chronic atrophy of the intestinal tract also months after treatment is terminated (Berthrong and Fajardo, 1981). The chronical absorption impairment of fats, carbohydrates, protein, bile salts, and electrolytes can lead to malabsorption and diarrhea as well as bacterial overgrowth and ultimately dysbiosis (Wedlake et al., 2008).

Chemotherapy-associated gastrointestinal toxicity is common in cancer patients and diarrhea is the most frequent clinical manifestation. Diarrhea is a known dose-limiting toxicity for cytotoxic agents like fluoropyrimidines (particularly fluorouracile and capecitabine) and irinotecan especially when these are used in association (Lee et al., 2014). Moreover, several targeted therapies, like tyrosine kinase inhibitors and monoclonal antibodies, are associated with diarrhea and more recently immune checkpoint inhibitors have shown to be also responsible for this adverse event (Cramer and Bresalier, 2017).

The rapidly dividing enterocytes are critically affected by common cytotoxic agents which increase apoptosis of epithelial cells in the intestinal mucosa followed by morphometrical changes and finally resulting in the hypoplastic atrophy of small intestine mucosa (Keefe et al., 2000).

These alterations of small bowel lining results in a combination of secretory and osmotic diarrhea (Parnes et al., 1994; Gibson and Stringer, 2009). Moreover, irinotecan and fluoropyrimidines undergo enzymatic modification in liver and gut, therefore gene polymorphisms can greatly affect the availability of the active compounds affecting the toxic effects of these drugs (Stein et al., 2010). Lastly, irinotecan is able to alter the gut microbiota favoring the proliferation of different genera of bacteria. The increased presence of bacterial $\beta$-glucuronidase determines increased level of the active metabolite of irinotecan causing significant damage and diarrhea (Stringer et al., 2007). 


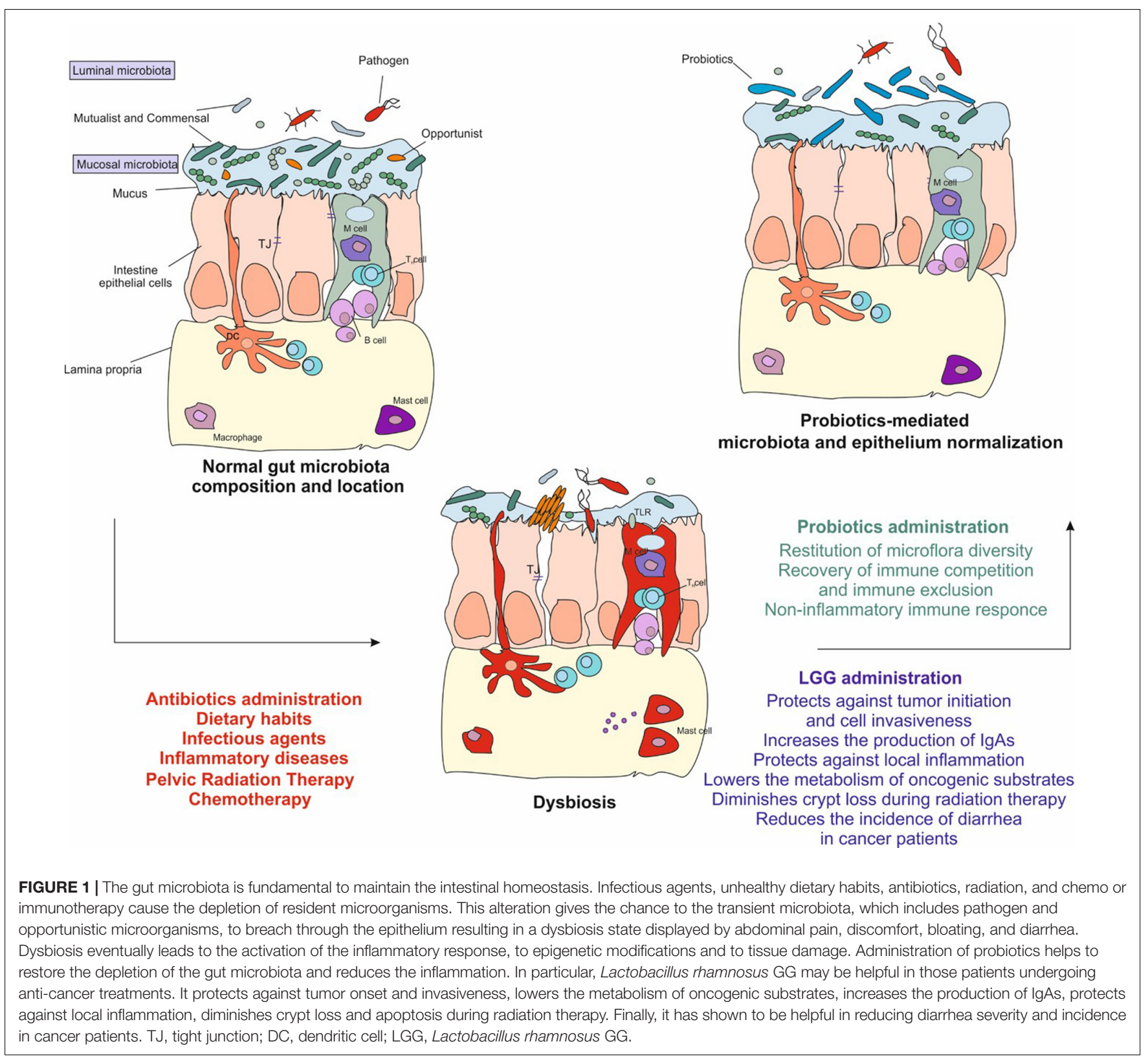

Up to $50 \%$ of patients treated with tyrosine kinase inhibitors develop diarrhea and this is sustained by several mechanisms not always completely elucidated (Keefe and Anthony, 2008; Hirsh et al., 2014). In the case of epidermal growth factor receptor (EGFR) TKIs mediated diarrhea, the inhibition of the EGFR pathway in the intestinal epithelium increases chloride secretion in the intestinal lumen mediated by an increase of intracellular cAMP (Uribe et al., 1996; Kleizen et al., 2000). Therefore, following the electric potential, sodium ions are pulled into the lumen along with water and this abnormal amount of electrolytes impair the physiological absorption of water causing secretory diarrhea.

Immune checkpoint inhibitors directed against CTLA-4 and PD-1 or PD-L1 molecules are also associated with diarrhea.
These drugs work by unleashing the immune response to tumor antigens but this can results in decreased tolerance to self antigens leading to inflammatory infiltration of normal tissues including the intestinal tract (Arriola et al., 2016). Clinically, immune checkpoints-induced colitis can range from mild diarrhea to severe colitis with bowel perforation.

An appropriate diet and probiotics administration can prevent and cure dysbiosis, restoring the correct equilibrium of gut microbiota (Delia et al., 2007; Osterlund et al., 2007; Spiller, 2008; Fuccio et al., 2009; Demers et al., 2014).

It was hypothesized that altered gut microbiota promotes and enhances cancer development, especially that of the intestinal tract (Tjalsma et al., 2012). The mechanisms, supporting this hypothesis, are described in several in vitro and in vivo studies 
(Tjalsma et al., 2012). Such mechanisms include the production of toxic metabolites that can lead to a chronic inflammatory condition, as well as the biosynthesis of genotoxic compounds interfering with cell cycle control or causing a direct DNA damage through the metabolism of dietary heterocyclic amines (Candela et al., 2011). Of note, chronic inflammation is associated with tumor development. Indeed, the nuclear transcription factor NF- $\kappa B$, which regulate several pro-inflammatory cytokines, is overexpressed in several cancer types (Mantovani, 2010).

Recently, preclinical and clinical evidences have linked the gut microbiota composition to specific response and toxicity patterns to immune checkpoint inhibitors treatments (Sivan et al., 2015; Vetizou et al., 2015; Chaput et al., 2017; Derosa et al., 2017). In particular, in a melanoma mouse model administration of commensal probiotics has shown to improve tumor control alone and in association with PD-L1 blockade; on the other hand tumors in antibiotic-treated or germ-free mice did not respond to CTLA-4 blockade (Sivan et al., 2015; Vetizou et al., 2015). These evidences have been related to an increased tumor-specific T-cell intervention and intratumoral CD8 + T-cell recruitment in mice with favorable commensal microbiota. Moreover, patients with metastatic renal cell carcinoma who received anti-PD-1 or PD-L1 monotherapy, or the combination of anti-PD-1 plus antiCTLA- 4 inhibitors, and underwent antibiotic therapy the month ahead the immunotherapy treatment, experience lower response rates to immune checkpoint inhibitors (Derosa et al., 2017). In melanoma patients specific gut microbiota patterns have been recently linked to improved response rate to ipilimumab (CTLA-4 inhibitor) but also to an increased occurrence of ipilimumab-induced colitis (Chaput et al., 2017). Taken together these findings support the hypothesis that preventive probiotics treatments may modulate cancer immunotherapy efficacy as well as the impact of specific toxicities and can be associated with the improvement of response rate in cancer patients.

Lactobacillus strains are LAB that are widespread in the environment and present in several surface, such as soil, water, and plants. Furthermore, Lactobacillus spp. are indigenous commensal inhabitants of the oral cavity, the GIT, and the female genital tract (Coudeyras et al., 2008).

To the best of our knowledge, Lactobacillus rhamnosus GG (LGG) (ATCC 53103) is one of the first probiotic studied in cancer and currently used in experimental designs (Goldin et al., 1996). The nomenclature of the species derives from the ability of LGG to metabolize and ferment rhamnose, a biochemical characteristic that is used to identify this species of Lactobacillus. To date, Lactobacillus spp. are identificated through several methods such as species-specific PCR, PFGE profiling of L. rhamnosus GG, bacteriophage typing, proteome analysis using 2D-DIGE and culture methods (i.e., using MRS growth agar medium) (Salminen et al., 2002, 2004, 2006; Koskenniemi et al., 2009; Villion and Moineau, 2009).

Some L. rhamnosus strains have beneficial effects on the organism as to be considered probiotics. In particular, LGG is able to withstand gastric acidity and bile salts effectively adhering to the gastrointestinal mucosa. The ability to resist gastric acidity and bile salts is a consequence of the ability of the bacterium to produce anti-stress proteins that give it greater survival capacity in intestinal transit after oral intake (Conway et al., 1987; Goldin et al., 1992). Adherence to the intestinal wall is also favored by the presence on the bacterial wall of exopolysaccharides rich in galactose residues and the presence of specific adhesive pili (Kankainen et al., 2009; Lebeer et al., 2009).

The effect on the immune system is explained by the stimulation and production of different cytokines such as TNF- $\alpha$, IL-1 $\beta$, IL-6, IL-10, IL-12, IFN- $\gamma$ and a particular protein, $\mathrm{p} 40$, secreted by $L$. rhamnosus GG cells that can reduce the inflammatory state and apoptosis of intestinal epithelial cells (Claes et al., 2012). Other apoptotic inhibition mechanisms are related to the modulation of cyclooxygenase-2 (COX-2), as will be described later in the manuscript (Korhonen et al., 2004; Ciorba et al., 2012).

Therefore, LGG is well characterized and it is known to have several anti-inflammatory effects (Khailova et al., 2016). Accordingly, treatment with LGG in animal models may reduce the risk of colon cancer through the modulation of the gut microbiota and the downregulation of pro-inflammatory molecules (Gamallat et al., 2016). LGG was first isolated in the late ' 80 from fecal sample of healthy adult (Gorbach, 1996) and subsequently its whole-genome sequence has been well characterized and registered (Morita et al., 2009). In particular, through the comparison of the genome sequence of LGG and other Lactobacillus spp., Kankainen et al. (2009) identified LGG-specific islands containing genes coding for three secreted LPXTG-like pilins (spaCBA) and a pilin-dedicated sortase notoriously involved in the mechanisms of adhesion to the intestinal mucosa (Kankainen et al., 2009). It matches the selection criteria for probiotics, including high adhesion in vitro, survival trough GIT (gastric acid, bile) and tendency to form colonies with a good persistence in the gut (Gorbach, 1996).

This review summarizes the most relevant preclinical studies describing the effects of LGG on cancer cells proliferation and tumor invasion. Furthermore, its safety in the clinical setting is here described. Table 1 summarizes the most important studies describing the role of LGG in cancer. Although further clinical studies are needed to better confirm the role of LGG in cancer, we can argue that LGG may be effective in preventing diarrhea during anti-cancer treatments and in increasing the patient compliance to therapy.

\section{LGG IN CANCER: IN VITRO STUDIES}

Several in vitro studies have been carried out to test the usefulness of $L$. rhamnosus GG in anti-cancer therapeutic practice and have focused more on the effects of this bacterial strain on metabolism, cell proliferation and invasiveness, and immunomodulation.

\section{Metabolism}

One of the uncovered effects of the L. rhamnosus GG is on the polyamines metabolism. Polyamines are positively charged molecules playing a major role in DNA stabilization and cellular growth (Larqué et al., 2007); the uncontrolled cell replication leads eventually to lose the homeostatic concentration of these compounds, thus reaping cancer development. Gastric 
TABLE 1 | Effects of Lactobacillus rhamnosus GG (LGG) in cancer-related intestinal disorders.

\begin{tabular}{|c|c|c|c|c|c|}
\hline \multirow[t]{2}{*}{ Disease } & \multicolumn{2}{|c|}{ Study model } & \multirow[t]{2}{*}{ Effect of LGG } & \multirow[t]{2}{*}{ Notes } & \multirow[t]{2}{*}{ Reference } \\
\hline & In vitro & In vivo & & & \\
\hline
\end{tabular}

Gastric carcinoma

HGC-27

AGS

Colon cancer

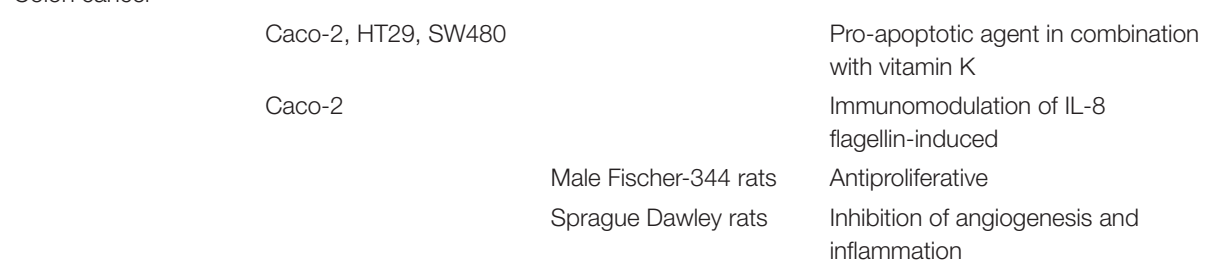

Metastatic CRC

HCT-116

Bladder cancer

Female C57BL/6 mice Tumor regression

Intestinal crypt loss

Inflammation

C57BL/6 mice

C57BL/6 mice
Reduction of the polyamine profile

Immunomodulation of IL-8

H. pyloridependent

Pro-apoptotic agent in combination

vitamin K

flagellin-induced

inflammation

Inhibition of invasiveness

Linsalata et al., 2010

$\begin{array}{ll}\text { DMH-induced } & \text { Goldin et al., } 1996 \\ \text { DMH-induced } & \text { Gamallat et al., } 2016\end{array}$

Rokka et al., 2008

Orlando et al., 2016

Lopez et al., 2008

Gamallat et al., 2016

Escamilla et al., 2012

Orthopic implantation Seow et al., 2010 of MB49

Radiation-induced

Ciorba et al., 2012

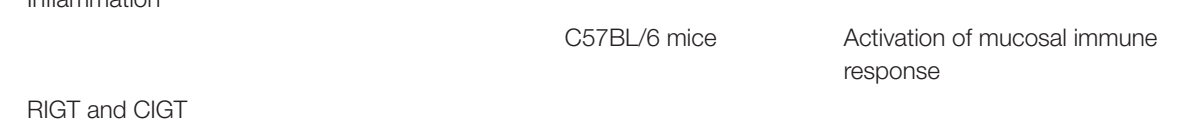

$15 \%$ lower incidence of diarrhea

Osterlund et al., 2007

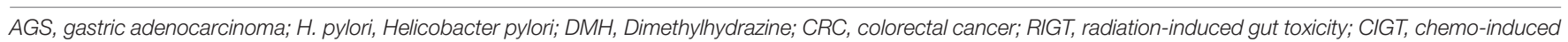
gut toxicity.

carcinoma cells (HGC-27) were exposed to LGG homogenate for 24 and $48 \mathrm{~h}$. The activity of the enzymes involved in this process was observed as well as the polyamines profile; the outcome was a dose-dependent decrease of the polyamine profile, up to the $20 \%$ in the $48 \mathrm{~h}$ and consecutively the detection of lower cell proliferation (Linsalata et al., 2010).

\section{Cell Proliferation and Invasiveness}

Vitamin $\mathrm{K} 1$ is believed to be an anti-proliferative agent; its administration in combination with viable LGG to three differently graded colon cancer cells (Caco-2, HT-29, and SW480) has shown a remarkable pro-apoptotic effect particularly on Caco- 2 cells at $48 \mathrm{~h}$ of treatment. This achievement suggests how the combination of agents can cooperate to extend one's functionality in reducing tumor expansion (Orlando et al., 2016). Invasiveness has been also investigated through the expression levels of matrix metalloproteinase-9 (MMP-9) and tight junction protein zona occludens-1 (ZO-1), as markers of the ECM degradation. Accordingly, metastatic colon cancer cells treated with cells free supernatants from LGG culture, achieved the gain of ZO-1 and the decrease of MMP-9 (Escamilla et al., 2012). These findings indicate an active role of the molecules released by LGG in the probiotic's growth medium. Such molecules may reduce the infiltration property of tumor cells and the invasive and metastatic potential of cancer cells.

\section{Immunomodulation}

The immunomodulating properties of LGG can be observed in a prospective use as a treatment, as well as a protective agent against inflammation. Neutrophils activation and dendritic cells (DC) maturation after exposure to LGG, have been explored by Cai et al. (2016), based on its suggested use against bladder cancer (Seow et al., 2010). In particular, neutrophils pre-cultured with LGG can stimulate the DCs maturation and the release of cytokines, like IL-12p70, which in turn activate the T-cells mediated immune response against the tumor environment. However, the optimal dose and time of LGG exposure is yet to be fully understood, because DCs seem to undergo exhaustion on high doses (Cai et al., 2016), thus reducing T-cells activation and the potential efficacy of the treatment. Moreover, LGG may be useful against chronic inflammation. The epithelium response to infective agents or to damages occurs also through the production of the pro-inflammatory chemokine IL-8. Of note, chronic inflammation induced by IL- 8 may cause cancer development (Waugh and Wilson, 2008). LGG has proven to be effective in lowering both the Helicobacter pylori-induced IL-8 production and its adhesion on gastric adenocarcinoma cells (Rokka et al., 2008). Flagellin, the principal protein component of bacterial flagellum, is able to increase pro-inflammatory chemokine levels, including IL-8, through nuclear translocation of $N F \kappa B$ (Lopez et al., 2008). In particular, flagellin can cause a 17 -fold increase in IL-8 production in Caco- 2 cells, whereas 
treatments with both viable LGG and UV-inactivated LGG inhibited the degradation of the I $\mathrm{I} B$. It, in turn, prevents the translocation of the transcription factor and the expression of IL-8 as an inflammatory messenger (Lopez et al., 2008). Other studies shown that peripheral blood mononuclear cells (PBMC) incubated in vitro with LAB, including LGG, showed a higher secretion of cytokines, proteins, or peptides acting as mediators and regulators of the immune response (Sheih et al., 2001), other results were an increase in the cytotoxic activity of natural killer cells against viruses and cancer cells (Delcenserie et al., 2008).

However, an in vitro study conducted on human DC and colon cancer cells (CaCo-2) treated with LGG did not highlight significant effect on inflammatory cytokine secretion. In particular, weaker non-significant effect was observed for IL-1b, IL-10, and TNFa, while no increase or decrement was observed for IL-12 secretion (Elmadfa et al., 2010).

\section{LGG IN CANCER: IN VIVO ANIMAL STUDIES}

\section{Protective Effects on Tumor Initiation}

The first in vivo study showed that L. rhamnosus GG can interfere with the tumor promotion of dimethyl-hydrazine (DMH) induced colon cancer in 344 male Fischer rats. In particular, the effect mediated by LGG was more evident in rats receiving a high fat regimen and pre-treated with LGG (Goldin et al., 1996), supporting the idea that this microorganism can achieve a protective role against colon cancer development by inhibiting or attenuating the mutagenic effects of carcinogenic substances.

Most recently, Gamallat et al. (2016) showed that LGG induced apoptosis and reduced the expression of several angiogenetic and inflammatory proteins in rats with $\mathrm{DMH}$ induced colon cancer. These data strongly support the notion that LGG administration may play a role in tumorigenesis prevention through angiogenesis and inflammation inhibition (Gamallat et al., 2016).

\section{Anti-inflammatory Effects during Anticancer Treatments}

Besides the preventive effects of LGG on tumor development and progression, several studies in animal models showed its efficacy in preventing radiation- and chemotherapy-induced toxicities (Seow et al., 2010; Ciorba et al., 2012; Wang et al., 2017). Ciorba et al. (2012) showed that the radiation-induced epithelial damage can be prevented by administration of LGG. In this study mice were treated with LGG 3 days before the beginning of radiation therapy and observed the changes in apoptosis and crypt survival. Apoptosis levels decreased from 30 to $17 \%$ in the LGG pretreated group while crypt survival doubled. The protective effects of LGG are mediated by the activation of the Toll-like receptor2 (TLR-2) and the shift of cycloxigenase-2 (COX-2) expression levels. Mesenchymal stem cells in the proliferative crypt region, express higher levels of COX-2 when treated with LGG (Ciorba et al., 2012). This may lead the release of elevated levels of prostaglandine-2 $\left(\mathrm{PGE}_{2}\right)$ which, in turn, may be associated with the epithelium proliferation against radiation damage (Riehl et al., 2000; Stenson, 2008; Ciorba et al., 2012).

It is known that LGG can modulate the inflammatory state occurring during cancer development and transformation. In particular, the inflammatory reduction is one of the most important goals for cancer prevention and treatment. Seow et al. (2010) showed that LGG may induce bladder cancer regression in mice with lower inflammatory toxicity compared to common anti-cancer treatments. This study's regimens consist in lyophilized LGG directly instilled in bladder, as well as Bacillus Calmette-Guerin (BCG) immunotherapy. Both treatments showed a considerable number of cured mice. However, in some cases the treatment with BCG showed an increased severe inflammatory response causing the delay of the scheduled treatment, whereas LGG did not cause any AEs (Seow et al., 2010), suggesting a protective role of LGG toward inflammation.

Intriguingly, Wang et al. (2017) recently described the antiinflammatory properties of LGG. The authors showed that the protein $\mathrm{p} 40$, an LGG-derived protein, was able to induce class switching of B-cell to IgA-secreting plasma cells in an EGFR-dependent manner (Wang et al., 2017). It is known that IgA secretion may reduce the inflammatory response by the promotion of toxins and pathogenic antigens clearance (Mantis et al., 2011). Accordingly, the lower inflammatory response may increase the adherence to the treatments and the compliance in cancer patients.

\section{CLINICAL EVIDENCES ON LGG ADMINISTRATION IN CANCER PATIENTS}

The most important effect of LGG, observed in cancers patients is linked to the reduction of acute or chronic diarrhea associated with chemotherapy and radiotherapy in cancer patients (Wang et al., 2016). Diarrhea affects the quality of life of patients as well as the anti-cancer treatment efficacy. Indeed, such patients experiencing diarrhea cannot tolerate the standard dose of therapy and need many adjustments in its dose and frequency. This side effect may also require hospitalization caused by the electrolytes loss. Acute or chronic diarrhea is observed among cancer patients treated with pelvic radiation therapy and/or with common antineoplastic agents. In detail, microvascular apoptosis and sclerosis of the vessels may occur during radiation therapy as results radiation-induced gut toxicity (RIGT) (Stansborough et al., 2017); while direct epithelial damage may be produced by chemotherapy (Van Sebille et al., 2015). Drugs used to stop diarrhea, such as loperamide, were not useful to restore the gut microflora. While the appropriate use of probiotics has been shown to be active and safe (Wang et al., 2016). For instance, Osterlund et al. (2007) reported interesting results in the phase III randomized study on cancer patients. The authors considered the combination of radio- and chemo-therapy and radiation therapy alone. The LGG was administered as 1-2 capsules/die $\left(10^{10}\right.$ Colony Forming Units) for 24 weeks during any anti-cancer treatment. The reports for diarrhea episode of grades 3 and 4 
were 15\% lower for those receiving LGG than the control group, suggesting that LGG supplementation may be a well-tolerated product able to ameliorate the compliance to the common cancer therapies (Osterlund et al., 2007).

Recently a meta-analysis of 11 randomized and placebo controlled studies evaluating the efficacy and safety of probiotics for prevention of chemo- and radiotherapy-induced diarrhea in people with abdominal and pelvic cancer was published. The study reported a potential beneficial effect of probiotics in the prevention of chemoradiotherapy-induced diarrhea with rare AEs (Wang et al., 2016).

\section{SAFETY}

After decades of administration of probiotics in clinical practice, some concerns on their safety have arisen due to the exponentially increased use and availability.

To best of our knowledge, the last guideline, reporting the potential side effects of probiotics, was described by the Joint FAO/WHO Working Group in 2002 (FAO/WHO, 2002). Systemic infections, deleterious metabolic activities, excessive immune stimulation in susceptible individuals and gene transfer were hypothesized. In particular, the report recommends the assessment of probiotics health claims and their safety use in a benefit patient's prospective ( $\mathrm{FAO} / \mathrm{WHO}$, 2002).

Many scientific studies have shown the therapeutic safety of the administration of probiotics, especially LGG. However, some studies have been reported bacteremia due to LGG overexpression, although these studies take into account particular classes of individuals such as children or diabetic patients (Land et al., 2005; Zein et al., 2008).

For a better understanding of the potential and rare AEs related to the consumption of probiotics, it is crucial to focus on their strain-by-strain characteristics (Marco et al., 2006; Shanahan, 2012). Indeed, it was observed that phenotypical differences within the same species, such as Lactobacillus rhamnosus, and different strain might be related to the behavioral switch from symbiotic to opportunistic L. rhamnosus, isolated from bacteremia, compared to the commercial Lactobacillus GG (ATCC 53103) (Ouwehand et al., 2004). However, such switch from symbiotic to opportunistic species is not yet assessed. Therefore, as reported below, a deep study of clinical records and/or case reports published in the literature remains the best approach to finding out any adverse effects of probiotics, such as LGG.

\section{LGG Administration in Healthy Subjects}

In a 5-year follow-up study, healthy-term infants, previously chosen for a double-blind controlled multicenter study, received LGG during the 1st year of age (Scalabrin et al., 2009). No serious $\mathrm{AE}$ correlated to the early consumption of LGG were described at the follow-up (Scalabrin et al., 2017). Intriguingly, it was shown that perinatal administration of LGG reduced allergy tendency in infants (Lundelin et al., 2017). Salminen et al. (2002) analyzed the effects of LGG consumption and lactobacilli bacteremia within 11-years period (1990-2000) in a large population. The study showed that only 11 cases were attributable to LGG and no time-related incidence was reported, deducing that no correlation between probiotic use of LGG and LGG bacteremia was observed (Salminen et al., 2002). Finally, no AEs after LGG consumption were observed among healthy elderly people, as reported in the study by Hibberd et al. (2014).

\section{LGG Administration in Cancer Patients}

To date, regarding the use of LGG in the oncology practice, there are no studies showing the onset of complications due to the administration of probiotics. With particular reference to oncologic pathologies, only two cases of bacteremia have been reported following the administration of probiotics. Both patients had onco-hematologic tumors and in both cases the development of bacteremia was attributable not to tumor but to bone marrow transplantation and to immunosuppressive therapies performed by patients (Majcher-Peszynska et al., 1999; Robin et al., 2010). These data show that the use of LGG in support of cancer therapy has a high level of safety, although very rarely were recorded episodes of bacteremia.

Redman et al. (2014) very nicely summarized the safety of probiotics combined with anti-cancer therapies. The authors analyzed 17 studies, including 1530 people (756 probiotics administered, 774 not consuming probiotics). The overlook for safety has been conducted considering different probiotics such as Saccharomyces boulardii, several species of Lactobacillus including LGG and Bifidobacterium together with Streptococcus thermophilus and VLS\#3 (a multispecies formula). Only rare AEs relate to probiotics administration were described but none of them was associated with the use of LGG even during neutropenia caused by chemotherapy (Redman et al., 2014).

\section{CONCLUSION}

Decades of clinical practice have experienced the use of probiotics as food supplies that can help patients, most likely affected by intestinal dysbiosis or by concomitant diseases. Since the time it was isolated, LGG has proven to be effective, through in vitro and in vivo experiments against inflammation, epithelial damage, invasiveness and proliferation of malignancies. Several studies on humans have been conducted to assess its usefulness against $\mathrm{AE}$ such diarrhea experienced by cancer patients. Even though the lack of studies homogeneity, the administration of LGG is clearly safe.

Well-designed clinical trials are mandatory to define the appropriate use of LGG during anti-cancer treatments, including pelvic radiation therapy and chemotherapy. According to the results obtained by such clinical trials, LGG may be regulated based on their use. We are confident that LGG may exert an important role in preserving the gut microbiota during such treatments and in improving the quality of life. Such positive effects of LGG administration may also enhance the adherence and compliance to treatments. 


\section{AUTHOR CONTRIBUTIONS}

GB, FT, FM, and FF critically contributed to elucidate the clinical aspects of the probiotics use in cancer therapy. MS contributed

\section{REFERENCES}

Andreyev, H. J., Davidson, S. E., Gillespie, C., Allum, W. H., Swarbrick, E., British Society of Gastroenterology, et al. (2012). Practice guidance on the management of acute and chronic gastrointestinal problems arising as a result of treatment for cancer. Gut 61, 179-192. doi: 10.1136/gutjnl-2011-300563

Arriola, E., Wheater, M., Lopez, M. A., Thomas, G., and Ottensmeiera, C. (2016). Evaluation of immune infiltration in the colonic mucosa of patients with ipilimumab-related colitis. Oncoimmunology 5:1209615. doi: 10.1080/ 2162402X.2016.1209615

Ashraf, R., and Shah, N. P. (2014). Immune system stimulation by probiotic microorganisms. Crit. Rev. Food Sci. Nutr. 54, 938-956. doi: 10.1080/10408398. 2011.619671

Banna, G. L., Collovà, E., Gebbia, V., Lipari, H., Giuffrida, P., Cavallaro, S., et al. (2010). Anticancer oral therapy: emerging related issues. Cancer Treat. Rev. 36, 595-605. doi: 10.1016/j.ctrv.2010.04.005

Benson, A. B., Ajani, J. A., Catalano, R. B., Engelking, C., Kornblau, S. M., Martenson, J. A. Jr., et al. (2004). Recommended guidelines for the treatment of cancer treatment-induced diarrhea. J. Clin. Oncol. 22, 2918-2926. doi: 10.1200/JCO.2004.04.132

Berthrong, M., and Fajardo, L. F. (1981). Radiation injury in surgical pathology. Part II. Alimentary tract. Am. J. Surg. Pathol. 5, 153-178. doi: 10.1097/ 00000478-198103000-00006

Cai, S., Kandasamy, M., Rahmat, J. N., Tham, S. M., Bay, B. H., Lee, Y. K., et al. (2016). Lactobacillus rhamnosus GG activation of dendritic cells and neutrophils depends on the dose and time of exposure. J. Immunol. Res. 2016:7402760. doi: 10.1155/2016/7402760

Candela, M., Guidotti, M., Fabbri, A., Brigidi, P., Franceschi, C., and Fiorentini, C. (2011). Human intestinal microbiota: cross-talk with the host and its potential role in colorectal cancer. Crit. Rev. Microbiol. 37, 1-14. doi: 10.3109/1040841X. 2010.501760

Chaput, N., Lepage, P., Coutzac, C., Soularue, E., Le Roux, K., Monot, C., et al. (2017). Baseline gut microbiota predicts clinical response and colitis in metastatic melanoma patients treated with ipilimumab. Ann. Oncol. 28, 1368-1379. doi: 10.1093/annonc/mdx108

Ciorba, M. A., Riehl, T. E., Rao, M. S., Moon, C., Ee, X., Nava, G. M., et al (2012). Lactobacillus probiotic protects intestinal epithelium from radiation injury in a TLR-2/cyclo-oxygenase-2-dependent manner. Gut 61, 829-838. doi: 10.1136/gutjnl-2011-300367

Claes, I. J., Schoofs, G., Regulski, K., Courtin, P., Chapot-Chartier, M. P., Rolain, T., et al. (2012). Genetic and biochemical characterization of the cell wall hydrolase activity of the major secreted protein of Lactobacillus rhamnosus GG. PLoS ONE 7:e31588. doi: 10.1371/journal.pone.0031588

Conway, P. L., Gorbach, S. L., and Goldin, B. R. (1987). Survival of lactic acid bacteria in the human stomach and adhesion to intestinal cells. J. Dairy Sci. 70, 1-12. doi: 10.3168/jds.S0022-0302(87)79974-3

Coudeyras, S., Marchandin, H., Fajon, C., and Forestier, C. (2008). Taxonomic and strain-specific identification of the probiotic strain Lactobacillus rhamnosus 35 within the Lactobacillus casei group. Appl. Environ. Microbiol. 74, 2679-2689. doi: 10.1128/AEM.02286-07

Cramer, P., and Bresalier, R. S. (2017). Gastrointestinal and hepatic complications of immune checkpoint inhibitors. Curr. Gastroenterol. Rep. 19, 3. doi: 10.1007/ s11894-017-0540-6

Davis, C. D., and Milner, J. A. (2009). Gastrointestinal microflora, food components and colon cancer prevention. J. Nutr. Biochem. 20, 743-752. doi: 10.1016/j.jnutbio.2009.06.001

DeGruttola, A. K., Low, D., Mizoguchi, A., and Mizoguchi, E. (2016). Current understanding of dysbiosis in disease in human and animal models. Inflamm. Bowel Dis. 22, 1137-1150. doi: 10.1097/MIB.000000000 0000750 to discuss the characterization of LGG. RS, EC, LF, and ML discuss the role of LGG in pre-clinical studies. The manuscript was amended based on comments from all authors. All authors read and approved the final version of the manuscript.

Delcenserie, V., Martel, D., Lamoureux, M., Amiot, J., Boutin, Y., and Roy, D. (2008). Immunomodulatory effects of probiotics in the intestinal tract. Curr. Issues Mol. Biol. 10, 37-54.

Delia, P., Sansotta, G., Donato, V., Frosina, P., Messina, G., De Renzis, C., et al. (2007). Use of probiotics for prevention of radiation-induced diarrhea. World J. Gastroenterol. 13, 912-915. doi: 10.3748/wjg.v13.i6.912

Demers, M., Dagnault, A., and Desjardins, J. (2014). A randomized double-blind controlled trial: impact of probiotics on diarrhea in patients treated with pelvic radiation. Clin. Nutr. 33, 761-767. doi: 10.1016/j.clnu.2013.10.015

Denham, J. W., and Hauer-Jensen, M. (2002). The radiotherapeutic injury - a complex 'wound'. Radiother. Oncol. 63, 129-145. doi: 10.1016/S0167-8140(02) 00060-9

Derosa, L., Routy, B., Enot, D., Baciarello, G., Massard, C., Loriot, Y., et al (2017). Impact of antibiotics on outcome in patients with metastatic renal cell carcinoma treated with immune checkpoint inhibitors. Paper Presented at the Genitourinary Cancers Symposium, Orlando, FL. doi: 10.1200/JCO.2017.35.6_ suppl.462

Elmadfa, I., Klein, P., and Meyer, A. L. (2010). Immune-stimulating effects of lactic acid bacteria in vivo and in vitro. Proc. Nutr. Soc. 69, 416-420. doi: $10.1017 /$ S0029665110001710

Escamilla, J., Lane, M. A., and Maitin, V. (2012). Cell-free supernatants from probiotic Lactobacillus casei and Lactobacillus rhamnosus GG decrease colon cancer cell invasion in vitro. Nutr. Cancer 64, 871-878. doi: 10.1080/01635581. 2012.700758

FAO/WHO (2002). Joint Working Group. Guidelines for the Evaluation of Probiotics in Food. Geneva: WHO.

Fuccio, L., Guido, A., Eusebi, L. H., Laterza, L., Grilli, D., Cennamo, V., et al. (2009). Effects of probiotics for the prevention and treatment of radiationinduced diarrhea. J. Clin. Gastroenterol. 43, 506-513. doi: 10.1097/MCG. 0b013e3181a1f59c

Gamallat, Y., Meyiah, A., Kuugbee, E. D., Hago, A. M., Chiwala, G., Awadasseid, A., et al. (2016). Lactobacillus rhamnosus induced epithelial cell apoptosis, ameliorates inflammation and prevents colon cancer development in an animal model. Biomed. Pharmacother. 83, 536-541. doi: 10.1016/j.biopha.2016.07.001

Gibson, R. J., and Stringer, A. M. (2009). Chemotherapy-induced diarrhoea. Curr. Opin. Support. Palliat. Care 3, 31-35. doi: 10.1097/SPC.0b013e32832531bb

Gill, H. S., Rutherfurd, K. J., Prasad, J., and Gopal, P. K. (2000). Enhancement of natural and acquired immunity by Lactobacillus rhamnosus (HN001), Lactobacillus acidophilus (HN017) and Bifidobacterium lactis (HN019). Br. J. Nutr. 83, 167-176. doi: 10.1017/S0007114500000210

Goldin, B. R., Gorbach, S. L., Saxelin, M., Barakat, S., Gualtieri, L., and Salminen, S. (1992). Survival of Lactobacillus species (strain GG) in human gastrointestinal tract. Dig. Dis. Sci. 37, 121-128. doi: 10.1007/BF01308354

Goldin, B. R., Gualtieri, L. J., and Moore, R. P. (1996). The effect of Lactobacillus GG on the initiation and promotion of DMH-induced intestinal tumors in the rat. Nutr. Cancer 25, 197-204. doi: 10.1080/01635589609514442

Gorbach, S. L. (1996). The discovery of Lactobacillus GG. Nutr. Today 31, 2S-5S. doi: 10.1097/00017285-199611001-00002

Guandalini, S. (2011). Probiotics for prevention and treatment of diarrhea. J. Clin. Gastroenterol. 45(Suppl.), S149-S153. doi: 10.1097/MCG.0b013e3182257e98

Hatoum, O. A., Otterson, M. F., Kopelman, D., Miura, H., Sukhotnik, I., Larsen, B. T., et al. (2006). Radiation induces endothelial dysfunction in murine intestinal arterioles via enhanced production of reactive oxygen species. Arterioscler. Thromb. Vasc. Biol. 26, 287-294. doi: 10.1161/01.ATV.0000198399. $40584.8 \mathrm{c}$

Hibberd, P. L., Kleimola, L., Fiorino, A. M., Botelho, C., Haverkamp, M., Andreyeva, I., et al. (2014). No evidence of harms of probiotic Lactobacillus rhamnosus GG ATCC 53103 in healthy elderly-a phase i open label study to assess safety, tolerability and cytokine responses. PLOS ONE 9:e113456. doi: 10.1371/journal.pone.0113456 
Hirsh, V., Blais, N., Burkes, R., Verma, S., and Croitoru, K. (2014). Management of diarrhea induced by epidermal growth factor receptor tyrosine kinase inhibitors. Curr. Oncol. 21, 329-336. doi: 10.3747/co.21.2241

Hullar, A. J. M., and Fu, B. C. (2014). Diet, the gut microbiome, and epigenetics. Cancer J. 20, 170-175. doi: 10.1097/PPO.0000000000000053

Kamada, N., Chen, G. Y., Inohara, N., and Núñez, G. (2013). Control of pathogens and pathobionts by the gut microbiota. Nat. Immunol. 14, 685-690. doi: $10.1038 /$ ni.2608

Kankainen, M., Paulin, L., Tynkkynen, S., von Ossowski, I., Reunanen, J., Partanen, P., et al. (2009). Comparative genomic analysis of Lactobacillus rhamnosus GG reveals pili containing a human- mucus binding protein. Proc. Natl. Acad. Sci. U.S.A. 106, 17193-17198. doi: 10.1073/pnas.090 8876106

Keefe, D., and Anthony, L. (2008). Tyrosine kinase inhibitors and gut toxicity: a new era in supportive care. Curr. Opin. Support. Palliat. Care 2, 19-21. doi: 10.1097/SPC.0b013e3282f5273f

Keefe, D. M. K., Brealey, J., Goland, G. J., and Cummins, A. G. (2000). Chemotherapy for cancer causes apoptosis that precedes hypoplasia in crypts of the small intestine in humans. Gut 47, 632-637. doi: 10.1136/gut.47.5.632

Khailova, L., Baird, C. H., Rush, A. A., Barnes, C., and Wischmeyer, P. E. (2016). Lactobacillus rhamnosus GG treatment improves intestinal permeability and modulates inflammatory response and homeostasis of spleen and colon in experimental model of Pseudomonas aeruginosa pneumonia. Clin. Nutr. doi: 10.1016/j.clnu.2016.09.025 [Epub ahead of print].

Kleizen, B., Braakman, I., and de Jonge, H. R. (2000). Regulated trafficking of the CFTR chloride channel. Eur. J. Cell Biol. 79, 544-556. doi: 10.1078/0171-933500078

Korhonen, R., Kosonen, O., Korpela, R., and Moilanen, E. (2004). The expression of COX2 protein induced by Lactobacillus rhamnosus GG, endotoxin and lipoteichoic acid in T84 epithelial cells. Lett. Appl. Microbiol. 39, 19-24. doi: 10.1111/j.1472-765X.2004.01531.x

Koskenniemi, K., Koponen, J., Kankainen, M., Savijoki, K., Tynkkynen, S., de Vos, W. M., et al. (2009). Proteome analysis of Lactobacillus rhamnosus GG using 2-D DIGE and mass spectrometry shows differential protein production in laboratory and industrial-type growth media. J. Proteome Res. 8, 4993-5007. doi: $10.1021 /$ pr9003823

Krautkramer, K. A., Kreznar, J. H., Romano, K. A., Vivas, E. I., Barrett-Wilt, G. A., Rabaglia, M. E., et al. (2016). Diet-microbiota interactions mediate global epigenetic programming in multiple host tissues. Mol. Cell 64, 982-992. doi: 10.1016/j.molcel.2016.10.025

Land, M. H., Rouster-Stevens, K., Woods, C. R., Cannon, M. L., Cnota, J., and Shetty, A. K. (2005). Lactobacillus sepsis associated with probiotic therapy. Pediatrics 115, 178-181. doi: 10.1542/peds.2004-2137

Larqué, E., Sabater-Molina, M., and Zamora, S. (2007). Biological significance of dietary polyamines. Nutrition 23, 87-95. doi: 10.1016/j.nut.2006.09.006

Lebeer, S., Verhoeven, T. L., Francius, G., Schoofs, G., Lambrichts, I., Dufrêne, Y., et al. (2009). Identification of a gene cluster for the biosynthesis of a long, galactose-rich exopolysaccharide in Lactobacillus rhamnosus GG and functional analysis of the priming glycosyltransferase. Appl. Environ. Microbiol. 75, 3554-3563. doi: 10.1128/AEM.02919-08

Lee, C. S., Ryan, E. J., and Doherty, G. A. (2014). Gastro-intestinal toxicity of chemotherapeutics in colorectal cancer: the role of inflammation. World J. Gastroenterol. 20, 3751-3761. doi: 10.3748/wjg.v20.i14.3751

Ley, R. E., Peterson, D. A., and Gordon, J. I. (2006). Ecological and evolutionary forces shaping microbial diversity in the human intestine. Cell 124, 837-848. doi: 10.1016/j.cell.2006.02.017

Lin, L., and Zhang, J. (2017). Role of intestinal microbiota and metabolites on gut homeostasis and human diseases. BMC Immunol. 18:2. doi: 10.1186/s12865016-0187-3

Linsalata, M., Cavallini, A., Messa, C., Orlando, A., Refolo, M. G., and Russo, F. (2010). Lactobacillus rhamnosus GG influences polyamine metabolism in HGC-27 gastric cancer cell line: a strategy toward nutritional approach to chemoprevention of gastric cancer. Curr. Pharm. Des. 16, 847-853. doi: 10.2174/138161210790883598

Lopez, M., Li, N., Kataria, J., Russell, M., and Neu, J. (2008). Live and ultravioletinactivated Lactobacillus rhamnosus GG decrease flagellin-induced interleukin8 production in Caco-2 cells. J. Nutr. 138, 2264-2268. doi: 10.3945/jn.108. 093658
Lundelin, K., Poussa, T., Salminen, S., and Isolauri, E. (2017). Long-term safety and efficacy of perinatal probiotic intervention: evidence from a follow-up study of four randomized, double-blind, placebo-controlled trials. Pediatr. Allergy Immunol. 28, 170-175. doi: 10.1111/pai.12675

Majcher-Peszynska, J., Heine, W., Richter, I., Eggers, G., and Mohr, C. (1999). Persistent Lactobacillus casei subspecies rhamnosus bacteremia in a 14 year old girl with acute myeloid leukemia. A case report. Klin. Padiatr. 211, 53-56. doi: 10.1055/s-2008-1043764

Mantis, N. J., Rol, N., and Corthésy, B. (2011). Secretory IgA's complex roles in immunity and mucosal homeostasis in the gut. Mucosal Immunol. 4, 603-611. doi: $10.1038 / \mathrm{mi} .2011 .41$

Mantovani, A. (2010). Molecular pathways linking inflammation and cancer. Curr. Mol. Med. 10, 369-373. doi: 10.2174/156652410791316968

Marco, M. L., Pavan, S., and Kleerebezem, M. (2006). Towards understanding molecular modes of probiotic action. Curr. Opin. Biotechnol. 17, 204-210. doi: 10.1016/j.copbio.2006.02.005

McQuade, R. M., Stojanovska, V., Abalo, R., Bornstein, J. C., and Nurgali, K. (2016). Chemotherapy-induced constipation and diarrhea: pathophysiology, current and emerging treatments. Front. Pharmacol. 7:414. doi: 10.3389/fphar.2016. 00414

Mego, M., Chovanec, J., Vochyanova-Andrezalova, I., Konkolovsky, P., Mikulova, M., Reckova, M., et al. (2015). Prevention of irinotecan induced diarrhea by probiotics: a randomized double blind, placebo controlled pilot study. Complement. Ther. Med. 23, 356-362. doi: 10.1016/j.ctim.2015.03.008

Morita, H., Toh, H., Oshima, K., Murakami, M., Taylor, T. D., Igimi, S., et al. (2009). Complete genome sequence of the probiotic Lactobacillus rhamnosus ATCC 53103. J. Bacteriol. 191, 7630-7631. doi: 10.1128/JB.01287-09

Orlando, A., Linsalata, M., and Russo, F. (2016). Antiproliferative effects on colon adenocarcinoma cells induced by co-administration of vitamin $\mathrm{K} 1$ and Lactobacillus rhamnosus GG. Int. J. Oncol. 48, 2629-2638. doi: 10.3892/ijo.2016. 3463

Osterlund, P., Ruotsalainen, T., Korpela, R., Saxelin, M., Ollus, A., Valta, P., et al. (2007). Lactobacillus supplementation for diarrhoea related to chemotherapy of colorectal cancer: a randomised study. Br. J. Cancer 97, 1028-1034. doi: $10.1038 /$ sj.bjc. 6603990

Ouwehand, A. C., Saxelin, M., and Salminen, S. (2004). Phenotypic differences between commercial Lactobacillus rhamnosus GG and L. rhamnosus strains recovered from blood. Clin. Infect. Dis. 39, 1858-1860. doi: 10.1086/425741

Pace, F., Pace, M., and Quartarone, G. (2015). Probiotics in digestive diseases: focus on Lactobacillus GG. Minerva Gastroenterol. Dietol. 61, 273-292.

Parnes, H. L., Fung, E., and Schiffer, C. A. (1994). Chemotherapy-induced lactose intolerance in adults. Cancer 74, 1629-1633. doi: 10.1002/1097-0142(19940901) 74:5<1629::AID-CNCR2820740523>3.0.CO;2-L

Redman, M. G., Ward, E. J., and Phillips, R. S. (2014). The efficacy and safety of probiotics in people with cancer: a systematic review. Ann. Oncol. 25, 1919-1929. doi: 10.1093/annonc/mdu106

Riehl, T., Cohn, S., Tessner, T., Schloemann, S., and Stenson, W. F. (2000). Lipopolysaccharide is radioprotective in the mouse intestine through a prostaglandin-mediated mechanism. Gastroenterology 118, 1106-1116. doi: 10.1016/S0016-5085(00)70363-5

Robin, F., Paillard, C., Marchandin, H., Demeocq, F., Bonnet, R., and Hennequin, C. (2010). Lactobacillus rhamnosus meningitis following recurrent episodes of bacteremia in a child undergoing allogeneic hematopoietic stem cell transplantation. J. Clin. Microbiol. 48, 4317-4319. doi: 10.1128/JCM.00250-10

Rokka, S., Myllykangas, S., and Joutsjoki, V. (2008). Effect of specific colostral antibodies and selected lactobacilli on the adhesion of Helicobacter pylori on AGS cells and the Helicobacter-induced IL-8 production. Scand. J. Immunol. 68, 280-286. doi: 10.1111/j.1365-3083.2008.02138.x

Salminen, M. K., Rautelin, H., Tynkkynen, S., Poussa, T., Saxelin, M., Valtonen, V., et al. (2004). Lactobacillus bacteremia, clinical significance, and patient outcome, with special focus on probiotic L. rhamnosus GG. Clin. Infect. Dis. 38, 62-69. doi: 10.1086/380455

Salminen, M. K., Rautelin, H., Tynkkynen, S., Poussa, T., Saxelin, M., Valtonen, V., et al. (2006). Lactobacillus bacteremia, species identification, and antimicrobial susceptibility of 85 blood isolates. Clin. Infect. Dis. 42, e35-e44. doi: 10.1086/ 500214

Salminen, M. K., Tynkkynen, S., Rautelin, H., Saxelin, M., Vaara, M., Ruutu, P., et al. (2002). Lactobacillus bacteremia during a rapid increase in probiotic use 
of Lactobacillus rhamnosus GG in Finland. Clin. Infect. Dis. 35, 1155-1160. doi: $10.1086 / 342912$

Scalabrin, D., Harris, C., Johnston, W. H., and Berseth, C. L. (2017). Long-term safety assessment in children who received hydrolyzed protein formulas with Lactobacillus rhamnosus GG: a 5-year follow-up. Eur. J. Pediatr. 176, 217-224. doi: 10.1007/s00431-016-2825-4

Scalabrin, D. M., Johnston, W. H., Hoffman, D. R., P’Pool, V. L., Harris, C. L., and Mitmesser, S. H. (2009). Growth and tolerance of healthy term infants receiving hydrolyzed infant formulas supplemented with Lactobacillus rhamnosus GG: randomized, double-blind, controlled trial. Clin. Pediatr. (Phila). 48, 734-744. doi: 10.1177/0009922809332682

Sekirov, I., Russell, S. L., Antunes, L. C. M., and Finlay, B. B. (2010). Gut microbiota in health and disease. Physiol. Rev. 90, 859-904. doi: 10.1152/physrev.00045. 2009

Seow, S. W., Cai, S., Rahmat, J. N., Bay, B. H., Lee, Y. K., Chan, Y. H., et al. (2010). Lactobacillus rhamnosus GG induces tumor regression in mice bearing orthotopic bladder tumors. Cancer Sci. 101, 751-758. doi: 10.1111/j.1349-7006. 2009.01426.x

Shanahan, F. (2012). A commentary on the safety of probiotics. Gastroenterol. Clin. North Am. 41, 869-876. doi: 10.1016/j.gtc.2012.08.006

Sheih, Y. H., Chiang, B. L., Wang, L. H., Liao, C. K., and Gill, H. S. (2001). Systemic immunity-enhancing effects in healthy subjects following dietary consumption of the lactic acid bacterium Lactobacillus rhamnosus HN001. J. Am. Coll. Nutr. 20, 149-156. doi: 10.1080/07315724.2001.10719027

Siegel, R. L., Miller, K. D., and Jemal, A. (2017). Cancer statistics, 2017. CA Cancer J. Clin. 67, 7-30. doi: 10.3322/caac.21387

Sivan, A., Corrales, L., Hubert, N., Williams, J. B., Aquino-Michaels, K., Earley, Z. M., et al. (2015). Commensal Bifidobacterium promotes antitumor immunity and facilitates anti-PD-L1 efficacy. Science 350, 1084-1089. doi: 10.1126/ science.aac 4255

Spiller, R. (2008). Review article: probiotics and prebiotics in irritable bowel syndrome. Aliment. Pharmacol. Ther. 28, 385-396. doi: 10.1111/j.1365-2036. 2008.03750

Stansborough, R. L., Bateman, E. H., Al-Dasooqi, N., Bowen, J. M., Keefe, D. M. K., Yeoh, A. S. J., et al. (2017). Fractionated abdominal irradiation induces intestinal microvascular changes in an in vivo model of radiotherapy-induced gut toxicity. Support. Care Cancer 25, 1973-1983. doi: 10.1007/s00520-0173601-3

Stein, A., Voigt, W., and Jordan, K. (2010). Chemotherapy-induced diarrhea: pathophysiology, frequency and guideline-based management. Ther. Adv. Med. Oncol. 2, 51-63. doi: 10.1177/1758834009355164

Stenson, W. F. (2008). Toll-like receptors and intestinal epithelial repair. Curr. Opin. Gastroenterol. 24, 103-107. doi: 10.1097/MOG.0b013e3282f $44 \mathrm{a} 2 \mathrm{a}$

Stringer, A. M., Gibson, R. J., Logan, R. M., Bowen, J. M., Yeoh, A. S., Burns, J., et al. (2007). Chemotherapy-induced diarrhea is associated with changes in the luminal environment in the DA rat. Exp. Biol. Med. 232, 96-106.

The Human Microbiome Project Consortium (2012). Structure, function and diversity of the healthy human microbiome. Nature 486, 207-214. doi: 10.1038/ nature 11234
Tjalsma, H., Boleij, A., Marchesi, J. R., and Dutilh, B. E. (2012). A bacterial driverpassenger model for colorectal cancer: beyond the usual suspects. Nat. Rev. Microbiol. 10, 575-582. doi: 10.1038/nrmicro2819

Uribe, J. M., Gelbmann, C. M., Traynor-Kaplan, A. E., and Barrett, K. E. (1996). Epidermal growth factor inhibits $\mathrm{Ca}(2+)$-dependent $\mathrm{Cl}$ - transport in T84 human colonic epithelial cells. Am. J. Physiol. Cell Physiol. 271, C914-C922.

Van Sebille, Y. Z., Stansborough, R., Wardill, H. R., Bateman, E., Gibson, R. J., and Keefe, D. M. (2015). Management of mucositis during chemotherapy: from pathophysiology to pragmatic therapeutics. Curr. Oncol. Rep. 17, 50. doi: 10.1007/s11912-015-0474-9

Vetizou, M., Pitt, J. M., Daillere, R., Lepage, P., Waldschmitt, N., Flament, C., et al. (2015). Anticancer immunotherapy by CTLA-4 blockade relies on the gut microbiota. Science 350, 1079-1084. doi: 10.1126/science.aad1329

Villion, M., and Moineau, S. (2009). Bacteriophages of lactobacillus. Front. Biosci. (Landmark Ed). 14:1661-1683. doi: 10.2741/3332

Wang, Y., Liu, L., Moore, D. J., Shen, X., Peek, R. M., Acra, S. A., et al. (2017). An LGG-derived protein promotes IgA production through upregulation of APRIL expression in intestinal epithelial cells. Mucosal Immunol. 10, 373-384. doi: $10.1038 / \mathrm{mi} .2016 .57$

Wang, Y. H., Yao, N., Wei, K. K., Jiang, L., Hanif, S., Wang, Z. X., et al. (2016). The efficacy and safety of probiotics for prevention of chemoradiotherapy-induced diarrhea in people with abdominal and pelvic cancer: a systematic review and meta-analysis. Eur. J. Clin. Nutr. 70, 1246-1253. doi: 10.1038/ejcn.2016.102

Waugh, D. J., and Wilson, C. (2008). The interleukin-8 pathway in cancer. Clin. Cancer Res. 14, 6735-6741. doi: 10.1158/1078-0432.CCR-07-4843

Wedlake, L., Thomas, K., McGough, C., and Andreyev, H. J. (2008). Small bowel bacterial overgrowth and lactose intolerance during radical pelvic radiotherapy: an observational study. Eur. J. Cancer 44, 2212-2217. doi: 10.1016/j.ejca.2008. 07.018

Zein, E. F., Karaa, S., Chemaly, A., Saidi, I., Daou-Chahine, W., and Rohban, R. (2008). Lactobacillus rhamnosus septicemia in a diabetic patient associated with probiotic use: a case report. Ann. Biol. Clin. (Paris) 66, 195-198. doi: 10.1684/ abc. 2008.0210

Zoetendal, E. G., Rajilić-Stojanović, M., and de Vos, W. M. (2008). Highthroughput diversity and functionality analysis of the gastrointestinal tract microbiota. Gut 57, 1605-1615. doi: 10.1136/gut.2007.133603

Conflict of Interest Statement: ML is the PI of a research grant founded by Dicofarm Spa to his University Department.

The other authors declare that the research was conducted in the absence of any commercial or financial relationships that could be construed as a potential conflict of interest.

Copyright (C) 2017 Banna, Torino, Marletta, Santagati, Salemi, Cannarozzo, Falzone, Ferraù and Libra. This is an open-access article distributed under the terms of the Creative Commons Attribution License (CC BY). The use, distribution or reproduction in other forums is permitted, provided the original author (s) or licensor are credited and that the original publication in this journal is cited, in accordance with accepted academic practice. No use, distribution or reproduction is permitted which does not comply with these terms. 